\title{
Research Article \\ Study on Heart Rate and Energy Expenditure in College Sports Training Based on Multisensor Perception
}

\author{
Ge Liu (i) and Baiqing Liu \\ Zhongnan University of Economics and Law, Hubei Wuhan 430074, China \\ Correspondence should be addressed to Ge Liu; z0004485@zuel.edu.cn
}

Received 31 August 2021; Accepted 13 October 2021; Published 13 November 2021

Academic Editor: Gengxin Sun

Copyright $\odot 2021 \mathrm{Ge}$ Liu and Baiqing Liu. This is an open access article distributed under the Creative Commons Attribution License, which permits unrestricted use, distribution, and reproduction in any medium, provided the original work is properly cited.

\begin{abstract}
Heart rate is one of the important indices to calculate and evaluate the intensity and quality of motion. It can scientifically and objectively reflect the intensity level of momentum and exercise in the process of exercise. It is an important index of athletic strength and physical fitness of athletes in college sports training. This paper analyzes the basic metabolic model of the human body and energy supply and demand model and constructs a scientific energy consumption test model for special sports activity. An algorithm for heart rate detection and energy consumption based on acceleration data acquisition is proposed. Therefore, it is proposed to calculate motion acceleration using bone point data obtained from a portable telephone camera sensor for special sports activity and to calculate kinetic energy consumption through detection data. A model for evaluating the proposed algorithm is also established.
\end{abstract}

\section{Introduction}

In the past, heart rate measurement under exercise was carried out by using heart rate frequency band. This device needs to install conductive heart rate frequency band in the chest to collect ECG signals of users and analyze heart rate [1]. Now, in sports research in China, polar heartbeat band with high recognition is used [2]. Because the heart rate belt is inconvenient to wear and the price is high, the physical training in many colleges and universities is unbearable. With the popularity of people who value health and devices that can be installed, the number of people who want to record their exercise status and heart rate has increased. Many companies are developing corresponding products. These new products are almost all in the form of watches. These products exceed the limit of heartbeat band and greatly improve the comfort of users. Therefore, rapid development has been made in recent years. These devices generally use photoelectric volume pulse wave to measure heart rate, but the accuracy of heart rate measurement of these devices during exercise is not very good [3]. For example, like Hua- wei's HUAWEI Watch, when the arm is quiet, you can wait about 11 seconds to get the current heartbeat value. Static measurements are 2 BPM different from medical devices, but if the arm is shaken or swung in the test, the test will fail.

In addition, most of these watches have the function of motion state recognition, which can recognize various motion states such as running, walking, and rest [4]. The recognition of this motion state is mostly based on acceleration sensors. As an auxiliary function of heart rate meter, the recognition of action state is also the research content of this paper.

With the development of society, people's cognition of sports training has changed and new understanding. In colleges and universities, people have higher requirements for the effect of physical education teaching and training. After all, university is the last link and crucial link in personnel training. Having a healthy and ripped body requires creative thinking to strengthen students' physique in the process of exercise [5]. The rapid development of the Internet has brought many positive impacts on all aspects of people's lives. For example, we communicate more closely and frequently with foreign countries through the Internet, and we can shop and entertain 
without leaving home. However, people's travel activities, exercise, and face-to-face communication are becoming less and less, which leads to obvious lack of physical fitness and deterioration of physical fitness of college students in China [6].

In order to change this situation, we analyzed the situation and put forward Tabata training method in physical education with high-intensity interval training as the focus, hoping to further improve the physical fitness and health level of college students. In the scheme of strengthening national physical fitness by sports put forward by our country, the overall level of aerobics in colleges and universities needs to be improved, so it is necessary to strengthen teaching, analyze the present situation, and prepare for improving the overall level. Combined with big data, the teaching methods of microcourses are applied to bodybuilding gymnastics in colleges and universities. Teachers can collect materials from the Internet, make videos, and then assign tasks to students, so as to achieve the purpose of learning and integrating knowledge. In addition, there are public online courses to realize the training of talents in the form of sharing, which shows that big data thinking provides a new perspective and ideas for the construction of aerobics online open courses [7]. Researchers collected and analyzed the physical exercise data of students from two Russian universities. Similarly, through the study of three courses, there are differences in physical preparation and functional status between students in nonphysical education colleges and physical education colleges, which show that the index value of functional status is related to training and its dynamic change is characterized by higher cost of adapting to physical functional status [8].

\section{Motion State Recognition Algorithm Based on Acceleration Signal}

\subsection{Feature Extraction}

2.1.1. Signal Preprocessing. Firstly, the acceleration signal is filtered. The original signal unit is converted to $9.8 \mathrm{~N} / \mathrm{kg}$ (gravity acceleration g), and a 30-point smoothing filter is adopted.

The short acceleration signal does not contain enough signal to recognize the action. Long-time signals cause serious delay to real-time systems [9]. According to experience, the shorter sliding window does not contain enough information to identify different actions, and the sliding time window is long, which is not suitable for real-time systems. After repeated experiments, the data every $8 \mathrm{~s}$ is finally selected to form a feature matrix for motion state recognition [10]. The formula of the sum vector is as follows:

$$
G=\sqrt{x^{2}+y^{2}+z^{2}}
$$

where $x, y$, and $z$ are the components of acceleration in three directions, respectively.

Here, signal processing is to make use of systematic errors or noises produced by monitoring equipment in data acquisition. These signals need to be processed in normal data, which leads to large errors in later data processing. In this paper, filters are used for filtering, and some signals are adjusted to slide windows to reduce errors.

\subsubsection{Time Domain Characteristics}

(1) Standard Deviation. In motion state recognition, the time domain features are mainly mean, standard deviation, energy, sample entropy, peak degree, deviation, quartile difference, and correlation coefficient. Some literatures show that the deviation in the upper and lower directions can effectively distinguish the next layer. Kurtosis in front and back can effectively distinguish running. Coefficients in the left and right directions can distinguish between walking and going up.

The original signal is set as $x(1), x(2), x(3), \cdots, x(n)$ the mean value of $x(n)$ signals is, and the standard deviation is defined as

$$
\delta=\sqrt{\frac{\sum_{i=1}^{n}\left(x_{i}-\bar{x}\right)^{2}}{n},} \quad i=1,2, \cdots, n .
$$

In formula (2), $n$ is the number of samples. The peak value in vertical direction is a time domain characteristic with good recognition effect.

(2) Sample Entropy. Sampling entropy is a method to detect the complexity of new time series. The larger the sampling entropy, the more complex the sequence and the worse the periodicity. The acceleration signal of human body motion has certain periodicity and unstable time series. Therefore, the sample entropy is suitable for being used as the eigenvalue in human operation state recognition. As shown in formula (3), the sample entropy calculation formula $n$ is the length of the sequence, and $m$ is the dimension.

Let the original signals be $X(1), X(2), X(3), \cdots, X(n)$, from which $M$ numbers are continuously extracted to form a group of $M$-dimensional vectors. $y_{m}(i)$ represents a vector composed of $m$ consecutive values starting from the $i$-th point.

$$
y_{m}(i)=\{x(i), x(i+1), x(i+m-1)\} \text {. }
$$

The distance between $y_{m}(i)$ and $y_{m}(j)$ is defined as $d[$ $\left.\left.y_{m}(i), y_{m}(j)\right)\right]$, and given the threshold $r$, the number of $i$ $\leq n-m$ is calculated for each value $\left.d\left[y_{m}(i), y_{m}(j)\right)\right]<r$. And the ratio of this number to the total distance is $n-m$ -1 , which is defined as

$$
B_{i}^{m}=\frac{\left.\sum_{j=1}^{n=m}\left\{\operatorname{num}\left(\mathrm{d}\left[y_{m}(i) \bigotimes y_{m}(j)\right)\right]<r\right)\right\}}{n-m-1} .
$$

Calculate its average for all $i$, add 1 to the dimension of $m$, and repeat the above steps to obtain

$$
\operatorname{SampEn}(m, r)=\lim _{n \longrightarrow \infty}\left\{-\ln \left[\frac{B^{m+1}(r)}{B^{m}(r)}\right]\right\} .
$$

Different values of $m, r$, and $n$ will get different sample entropy values. 
2.1.3. Frequency Domain Characteristics. For extracting feature vectors from human motion state recognition, many researches have been carried out using frequency domain features. The frequency domain features are FFT coefficients, DCT coefficients, spectral entropy, and energy spectral density. Comparing the performance of several frequency domain features, it is found that FFT coefficients have high resolution. Some scholars use PCA to reduce and reduce FFT coefficients and then have a significant impact on the accuracy of motion recognition. In the five exercises discussed in this section, FFT coefficients are applicable to the features identified as exercise states at the point that running, stepping, and walking cycles are good.

In this study, FFT coefficients are further analyzed, and it is suggested that the main frequency should be used as one of the eigenvalues. In this paper, the three-axis acceleration signals are converted by FFT, and the main frequency is extracted as the frequency domain feature. In this way, not only the high resolution of FFT is used but also the feature dimension is reduced and the computation is reduced.

\subsection{Classifier Design}

2.2.1. SVM Classifier. The basic idea of support vector machine is to transform the input space into high-dimensional space by linear transformation and obtain the best linear classification surface in the new space. To use the SVM Toolbox, you must select the kernel function to use. The following are the representations of several general kernel functions.

Linear kernel:

$$
k(x, y)=x^{T} y+c .
$$

Polynomial kernel:

$$
k(x, y)=\left(a x^{T} y+c\right)^{d} .
$$

Radial basis function:

$$
k(x, y)=\exp \left(-\gamma\|x-y\|^{2}\right) .
$$

The radial basis function is a real function whose value depends only on the distance from the origin, that is, $\Phi(x)$ $=\Phi(\|x\|)$, or it can also be the distance to any point $c$, which is called the center point, that is, $\Phi(x, c)=\Phi(\|x-c\|)$. Any function $\Phi$ satisfying the characteristic $\Phi(x)=\Phi(\|x\|)$ is called radial basis function, and the standard one generally uses Euclidean distance (also called Euclidean radial basis function), although other distance functions are also possible. In the neural network structure, it can be used as the main function of full connection layer and ReLU layer. Using various eigenvalues extracted in the previous section, Professor Lin Zhiren of Taiwan developed LibSVM for data training.

2.2.2. Decision Tree Classifier. Decision tree turns complex multicategory problems into several simple classification problems to solve. The key to tree creation is how to select features in each node to create a simple tree. Motion state recognition based on decision tree is generally divided into two steps. The learning of training set is used to generate decision tree. The generated decision tree is used to identify the motion state.

The flow of decision tree is simple, and the complexity of algorithm is low. The general decision tree algorithms are CART, ID3, C4.5, CHAID, and so on. Mr. Algorithm makes complex trees and cuts them according to the results of cross-validation and test group validation. It has a very powerful statistical analysis function. In addition, the rules contained in the results after data processing become easy to understand. Therefore, classification regression tree is a good method to establish statistical analysis model for characteristic data. According to the research, the prediction tree constructed by CART model is more accurate than the algebraic mathematical prediction benchmark constructed by general statistical methods in many cases. The more complex the data and the more variables, the more obvious the advantages of the algorithm. In CART (Classification and Regression Tree) algorithm, the Gini index structure is used to determine the tree, and the Gini index is defined as

$$
\operatorname{Gini}(D)=1-\sum_{i=1}^{m} p_{i}^{2},
$$

where $m$ still denotes the number of classes $C$ in the data set $D, p_{i}$ denotes the probability that any record of $D$ belongs to $C$, and $p_{i}$ is defined as the ratio of the number of groups belonging to $C_{i}$ in $D$ to the total number of $D$. If all records belong to the same category, then $P_{1}=1$ and Gini $(D)=0$, in which case the purity is the lowest. For each attribute that enumerates a nonempty proper subset of the attribute, the Gini coefficient after the attribute $R$ is split which is taken

$$
\operatorname{Gini}_{R}(D)=\frac{\left|D_{1}\right|}{|D|} \operatorname{Gini}\left(D_{1}\right)+\frac{\left|D_{2}\right|}{|D|} \operatorname{Gini}\left(D_{2}\right) .
$$

$D_{1}$ is a nonempty proper subset of $D, D_{2}$ is the complete set of $D_{1}, D_{1}+D_{2}=D$, attribute $R$ has multiple proper subsets, that is, Gini $R(D)$ has multiple values, and the smallest value is selected as the Gini index of $R$.

$$
\Delta \operatorname{Gini}(R)=\operatorname{Gini}(D)-\operatorname{Gini}_{R}(D) .
$$

The maximum attribute of Gini $(R)$ increment is used as the best splitting characteristic. In order to prevent the decision tree from fitting the sample, it needs to be pruned. CART adopts price. In the pruning method of complexity, price refers to the misclassification rate of samples. Complexity refers to the number of leaf nodes of a tree and defines the value complexity of a tree.

$$
c c(t)=\alpha * \text { Leaf }_{t}+\frac{E}{N} .
$$

In equation (12), $N$ is the number of decision tree training samples, $E$ is the number of decision tree error samples, and Leaf ${ }_{t}$ is the number of leaves and parameters of the tree. 
$\alpha$ measures the relationship between price and complexity, indicating the relationship between the degree of complexity reduction of pruned trees and price. For $t$, cut down its subtree $s$ and obtain a new tree new ${ }_{t}$ to replace the best leaf node in $t$. The training data can be divided into $M$ by new than $t$, but the number of leaf points contained in new ${ }_{t}$ is less than $t$. Equate the price complexity after replacement.

$$
c c(t)=c c\left(\text { new }_{t}\right) \Rightarrow \alpha * \operatorname{Leaf}_{t}+\frac{E}{N} \Rightarrow \alpha=\frac{M}{N\left(\text { leaf }_{s}-1\right)}
$$

In formula (13), $M$ is the number of misdivided samples increased after replacing the $S$ subtree of $T$ with leaf nodes. leaf $_{s}$ is the number of leaf nodes of the subtree $s$. The pruning step: the first step: calculate the $\alpha$ value of each nonleaf node of the complete decision tree $T_{\text {max }}$; loop off the subtree with the smallest $\alpha$ value until the root node is left; a series of pruning trees $\left\{T_{0}, T_{1}, T_{2}, \cdots, T_{m}\right\}$ are obtained, where $T_{0}$ is the complete decision tree $T_{\max }$ and $T_{i+1}$ is the result of pruning $T_{i}$. Step 2: using independent pruning set (nontraining set) to evaluate $T_{i}$ in the first step to obtain the best pruning tree; standard error SE is shown in equation (14).

In formula (13), $M$ is the number of error samples added after the leaf node replaces the $T$ subtree. leaf $_{s}$ is the number of leaf nodes of subtree $s$. Pruning steps are as follows.

Step 1. Calculate the complete decision tree. The value of each nonleaf node: cyclic cutting is minimal. $\alpha$ preserves the subtree of values before the root node. A series of pruning trees $\left\{T_{0}, T_{1}, T_{2}, \cdots, T_{m}\right\}$ are obtained, where $T_{0}$ is the complete decision tree $T_{\max }$ and $T_{i+1}$ is the result of pruning $T_{i}$.

Step 2. Using independent pruning set (nontraining set) to evaluate $T_{i}$ in the first step to obtain the best pruning tree, standard error SE defined as

$$
\operatorname{SE}(E)=\sqrt{\frac{E^{6} *\left(N^{6}-E\right)}{N^{6}}}
$$

In formula (14), $N^{\prime}$ is the number of pruning sets, and $E_{i}$ is defined as the error fraction of tree $T_{i}$ to pruning sets.

$$
E^{\prime}=\min \left(E_{i}\right)
$$

Optimal pruning tree: $T$ best meets the following conditions and has the least number of nodes. The condition that needs to be met: $E \leq E^{\prime}+\mathrm{SE}\left(E^{\prime}\right)$.

\section{Establishment of Energy Consumption Model of Sports Activities}

The energy consumption of daily physical activity often cannot meet the total sports energy consumption demand of the balance of energy supply and demand of human body, so it is necessary to increase additional activity energy consumption [11]. At present, the common sports activities include running, skipping rope, swimming, and aerobic exercise. The common characteristics of these sports are planning, regularity, sports goals, etc. Therefore, this paper chooses four movements, namely, opening and closing jump, left and right small jump, skipping rope, and squat, which do not need additional fitness equipment and have lower requirements for sports environment. Compared with the commonly used acceleration sensor method, the skeleton point data collected by the mobile phone camera sensor can reflect the whole body motion trend, so the motion acceleration can be calculated by using the skeleton point data obtained by the mobile phone camera sensor in special sports activities.

Every movement completed by the human body depends on muscles and bones, and the work done by the body during exercise leads to energy consumption. A strand points out that there is a linear relationship between work, force product, and oxygen consumption for motion, and the force product is the integral of force and time, as shown in

$$
W=\int_{t_{2}}^{t_{1}} F d t
$$

where $F$ is the force acting on human muscles, according to Newton's second law:

$$
W=\int_{t_{2}}^{t_{1}} \operatorname{mad} t
$$

The differential of force product reflects the amount of activity per unit time, and the type and intensity of dipping and cutting are obtained by using the acceleration of movement. Under the changing trend of three dimensions of space, the energy consumption in a certain time is calculated by using the information of human motion intensity and time. Generally speaking, from the physical and mechanical point of view, researchers choose to fix the acceleration sensor at the hip joint because the hip joint is closer to the center of gravity of the human body. Compared with wearable devices, image recognition technology greatly reduces the invasion of motion and can simply and conveniently obtain the position of the human center of gravity and the change of acceleration value during human motion.

3.1. Data Acquisition of Bone Point and Center of Gravity. The bone point recognition algorithm adopts BlazePose algorithm in Blaze series algorithms released by Google. The algorithm is based on lightweight convolution neural network architecture, which not only pursues the implementation of mobile devices but also ensures high recognition accuracy. In the recognition process, the algorithm can provide up to 32 body key point information per frame, as shown in Figure 1. Running BlazePose algorithm on a mobile phone with Qualcomm Snapdragon 888 processor can provide 22 frames of bone point data per second [12], which is enough to meet the acquisition frequency requirements of acceleration data.

In the common calculation methods of center of gravity, the moment synthesis method needs to calculate the barycentric 


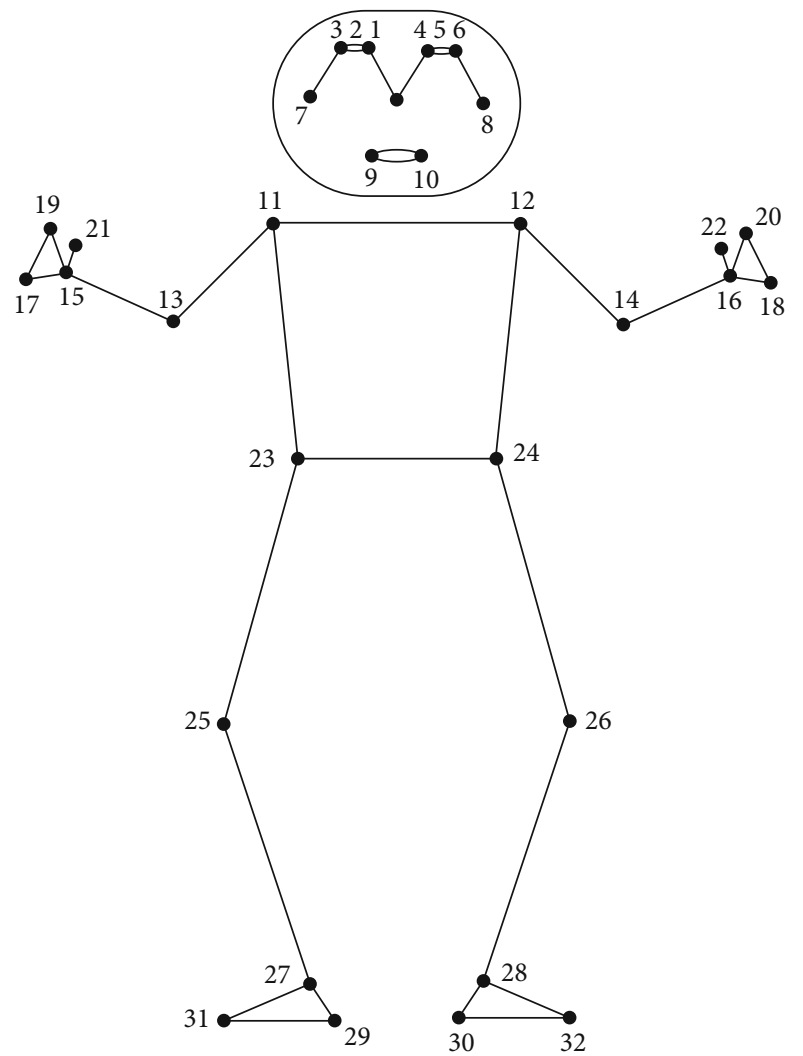

Figure 1: BlazePose algorithm bone point recognition diagram.

coordinates of the corresponding body parts by using the coordinates of bone points, while the multiplication coefficient method omits this step. On the basis of obtaining the coordinates of the center positions of each bone point of the body, it multiplies the corresponding bone point coefficient values and accumulates the results after multiplying each bone point to be the coordinates of the total barycentric of the human body. In this paper, the method proposed by Wei et al. is used to calculate the center of gravity of the human body in each frame image. The center of gravity is calculated according to the following equations:

$$
\begin{gathered}
X_{c}(t)=\sum_{i=1}^{15} k_{i}^{*} X_{i}(t), \\
Y_{c}(t)=\sum_{i=1}^{15} k_{i}^{*} Y_{i}(t) .
\end{gathered}
$$

In the formula, $X_{c}(t)$ and $Y_{c}(t)$ are the coordinates of bone point data obtained by BlazePose algorithm in $X$ and $Y$ axes, $X(t)$ and $Y_{i}(t)$ are the coordinates of a bone point $I$ in $X$ and $Y$ axes when the number of frames is $t$, and $k_{i}$ is the coefficient value corresponding to bone point $I$. The specific values are shown in Table 1.

Through the change trend of coordinate information of human skeleton points in each frame image, the displacement trend of human center of gravity in a period of movement is obtained by using the calculation method of center
TABLE 1: Human skeleton points and corresponding coefficients.

\begin{tabular}{lc}
\hline Bone point & Mapping coefficient value $(k)$ \\
\hline Nose & 0.0706 \\
Shoulder & 0.0356 \\
Midpoint of shoulder connection & 0.2391 \\
Elbow & 0.0580 \\
Hand & 0.0372 \\
Hip & 0.1297 \\
Midpoint of the line between hips & 0.1630 \\
Knee & 0.1630 \\
Distance & 0.0801 \\
\hline
\end{tabular}

of gravity, as shown in Figure 2, which shows the displacement trend of the center of gravity of left and right small jumps in a period of time.

3.2. Filtering of Bone Point Data. In actual use, the information of human bone points obtained by BlazePose algorithm has high accuracy, but there is still bone point shaking. In order to avoid the large calculation error of acceleration information caused by bone point jitter, which will affect the accuracy of energy consumption fitting experiment of special sports activities and the accuracy of energy consumption detection in actual use, it is necessary to filter the bone point data obtained by camera sensor.

Considering the real-time and effectiveness of the filtering algorithm, the same filtering algorithm as the daily physical activity energy consumption detection is selected in the energy consumption detection of special sports activities, that is, the moving average filtering algorithm. Figure 3 shows the filtering effect of barycentric coordinates in the process of human movement.

3.3. Acceleration Calculation Method. The data of 15 bone points in each frame obtained by BlazePose algorithm can calculate the coordinates of the center of gravity of the human body in each frame by multiplication coefficient method. During a period of movement, the coordinates of the center of gravity of the human body constitute a discrete displacement variable function, and the velocity variable and acceleration variable are expressed by the change of displacement and time during the movement, while the solution of velocity and acceleration needs to build a continuous displacement differential equation. In this paper, the finite difference method is used to solve this problem. Its principle is to use discrete functions instead of continuous variable functions and to replace differential equations with difference equations, thus establishing finite difference equations. The acceleration problem solved in this paper is a second-order differential problem, so the second-order central difference method is selected as the method to construct the difference shown in Figure 4.

First-order difference:

$$
\frac{\Delta f(x)}{\Delta x}=\frac{f(x+(h / 2))-f(x-(h / 2))}{h} .
$$



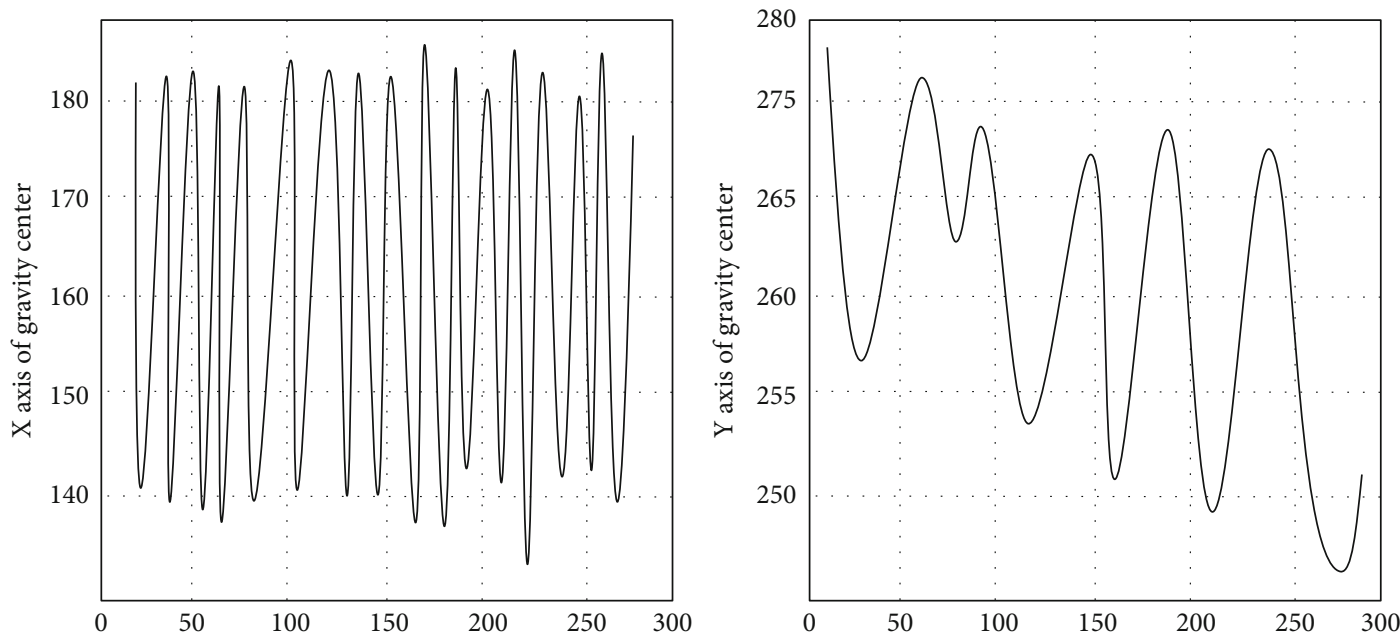

Figure 2: Relative displacement trend of left and right small jump center of gravity (a) $X$ axis and (b) $Y$ axis.
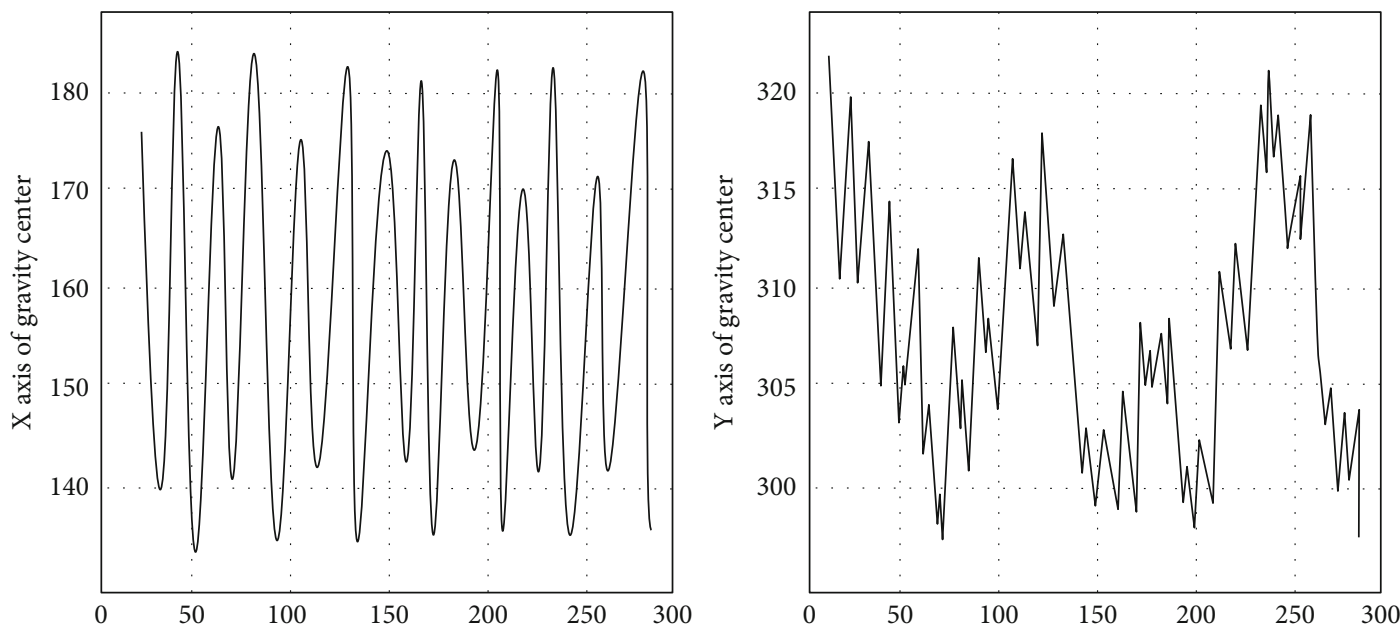

FIgURE 3: Filtering of (a) $X$ axis and (b) $Y$ axis of opening and closing jump center of gravity.
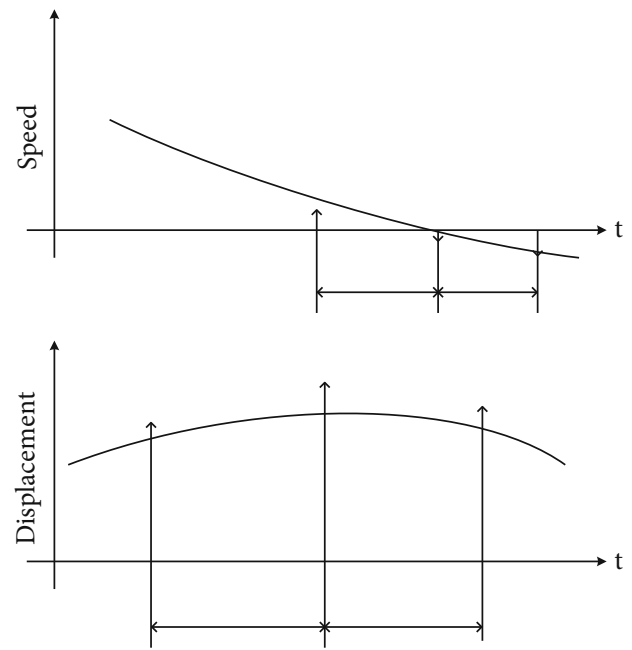

Figure 4: Schematic diagram of second-order central difference method.
Second-order difference:

$$
\frac{\Delta^{2} f(x)}{\Delta x^{2}}=\frac{f(x+h)-2 f(x)+f(x-h)}{h^{2}} .
$$

At the beginning of the movement, time $t=t_{0}$ :

$$
m \ddot{v}_{0}+c \dot{v}_{0}+k v_{0}=p_{0} .
$$

The initial acceleration is

$$
\ddot{v}_{0}=\frac{1}{m}\left(p_{0}-c \dot{v}_{0}-k v_{0}\right) \text {. }
$$

The velocity at the midpoint of the step before and after $t=t_{0}$ is

$$
\begin{aligned}
& \dot{v}_{-1 / 2}=\frac{v_{0}-v_{-1}}{h}, \\
& \dot{v}_{1 / 2}=\frac{v_{1}-v_{0}}{h},
\end{aligned}
$$


where $H$ is an equal time step and the acceleration in the middle of time is calculated by velocity:

$$
\ddot{v}_{0}=\frac{\dot{v}_{1 / 2}-\dot{v}_{-1 / 2}}{h}=\frac{1}{h^{2}}\left(v_{1}-v_{0}\right)-\frac{1}{h^{2}}\left(v_{0}-v_{-1}\right) \text {. }
$$

Thus, it is concluded that

$$
\ddot{v}_{0}=\frac{1}{h^{2}}\left(v_{1}-2 v_{0}\right)
$$

Eventually,

$$
v_{1}=v_{0}+h \dot{v}_{0}+\frac{h^{2}}{2 m}\left(p_{0}-c v_{0}-k v_{0}\right)
$$

The coordinates of bone points obtained by BlazePose algorithm are relative coordinates. The relative height coefficient at present time can be obtained by the ratio of the relative distance between the real height information of the human body and the bone point data of the human body in each frame image, and the displacement value multiplied by the current relative height coefficient when calculating the acceleration information in the motion process is the real motion displacement data. The acceleration value of the human body during training is calculated by using the finite difference method described above.

\subsection{Energy Consumption Calculation of Special Sports} Activities. The nutrients ingested by the human body meet the fixed ratio relationship in oxidation reaction: the ratio of reactant consumption to product production and released energy is constant and has nothing to do with the reaction steps and conditions. Fixed ratio relationship is the basis of indirect calorimetry. By detecting the consumption of sugar, fat, protein, and other nutrients in the human body during a period of time, the total energy produced in the human body during this period can be obtained by using the heat price of each nutrient.

According to the law of fixed ratio, researchers put forward another index to evaluate the heat price of food, which is called the oxygen heat price of food, which refers to the energy released when a certain food consumes $1 \mathrm{~L}$ oxygen in the oxidation reaction in vivo. Oxygen and heat price of food is a key index of energy detection. The key parameters of oxidation reaction of basic nutrients for human energy supply are shown in Table 2.

Different nutrients consume different $\mathrm{O}$ and produce different $\mathrm{CO}_{2}$ during oxidation reaction, and the energy produced is also different. The ratio of $\mathrm{CO}_{2}$ production to $\mathrm{O}_{2}$ consumption per unit time is called respiratory entropy, as shown in

$$
\text { Respiratory entropy }=\frac{\mathrm{Ml} \text { number of } \mathrm{CO}_{2} \text { produced }}{\mathrm{Ml} \text { number of } \mathrm{O}_{2} \text { consumed }}
$$

The respiratory entropy of sugar is 1 , and the oxygen thermal value provided by sugar is $21.1 \mathrm{~kJ} / \mathrm{L}$, that of fat is 0.71 , that of oxygen thermal value is $19.6 \mathrm{~kJ} / \mathrm{L}$, that of protein is 0.80 , and that of oxygen thermal value is $18.9 \mathrm{~kJ} / \mathrm{L}$. Under normal physiological conditions, most of the energy is produced by the reaction between sugar and fat, so in order to simplify the calculation method, the energy supply part of protein is ignored. According to the respiratory entropy, the reaction ratio of sugar and fat in this period of time was calculated, and the corresponding oxygen thermal valence was calculated.

\section{Experiment}

\subsection{Motion State Recognition Experiment}

4.1.1. Design of Exercise Experiment. According to the classification results of SCUT-NAA database, we propose an algorithm, which can effectively realize the recognition of motion state by using the acceleration sensor data of waist. However, the acceleration sensor of the heart rate meter is on the arm, and many literatures show that it is difficult to identify the motion state based on the acceleration sensor of the arm.

Firstly, this paper designs experiments and collects data. The subjects collected the sports data of 10 male college students in active service. First, they were required to stop for 1 minute, run for 3 minutes, rest for 1 minute, walk for 3 minutes, rest for 1 minute, stair for 2 minutes, rest for 1 minute, gymnastics for 2 minutes, and rest for 1 minute. All of them include five sports states: static, running, walking, jumping, and gymnastics.

4.1.2. Experimental Recognition Results and Analysis. The flowchart of multilevel motion recognition algorithm is designed by using decision tree classifier.

It can be seen from Tables 3 and 4 that the recognition rate of gymnastics is low. The main reason is that gymnastics has various sports states. When the human body does gymnastics, it is recognized as static, running, walking, etc., among which 21 are not recognized as static, which affects the overall recognition rate. There are two main reasons: (1) the gap between gymnastics and sports. (2) The feature values cannot better identify these five sports. These results show that the features extracted in the study cannot fully and effectively distinguish these sports states. The efficiency of the selected features needs to be further improved.

\subsection{Energy Consumption Test}

4.2.1. Experimental Design. Taking the energy consumption value detected by indirect calorimetry as the standard value and the front camera of mobile phone as the main tool, through the processing and calculation of the original image data, the energy consumption detection model is established on the basis of the recommended four sports and the validity of the equation is tested. The purpose is to establish a realtime, convenient, low-cost, and high-accuracy energy consumption detection method for special sports activities.

(1) Subjects. In this experiment, 45 subjects were selected. The body morphological indexes of the subjects are shown in Table 5. All the subjects were healthy and had no sports 
TABLe 2: Parameters of oxidation reaction of nutrients.

\begin{tabular}{lccccc}
\hline Nutrients & $\begin{array}{c}\text { Physiological heat value }(\mathrm{kJ} / \\
\mathrm{g})\end{array}$ & $\begin{array}{c}\mathrm{O}_{2} \text { consumption }(\mathrm{L} / \\
\mathrm{g})\end{array}$ & $\mathrm{CO}_{2}$ production $(\mathrm{L} /$ & $\begin{array}{c}\text { Oxygen thermal valence (kJ/ } \\
\text { g) }\end{array}$ & $\begin{array}{c}\text { Respiratory } \\
\text { entropy }\end{array}$ \\
\hline Sugar & 17.2 & 0.83 & 0.83 & 21.1 & 1 \\
Fat & 39.8 & 2.03 & 1.43 & 19.6 & 0.71 \\
Protein & 18.0 & 0.95 & 0.76 & 18.9 & 0.80 \\
\hline
\end{tabular}

TABLE 3: Recognition rate of motion experiment.

\begin{tabular}{lc}
\hline Kinds of sports & Recognition rate of action type \\
\hline Static & $79.41 \%$ \\
Running & $88.64 \%$ \\
Escape & $93.09 \%$ \\
Jump & $79.26 \%$ \\
Do gymnastics & $68.52 \%$ \\
Average & $81.78 \%$ \\
\hline
\end{tabular}

TABLE 4: Confusion matrix of exercise experiment.

\begin{tabular}{lccccc}
\hline Status & Static & Running & Escape & Jump & Do gymnastics \\
\hline Static & 536 & 29 & 24 & 45 & 41 \\
Running & 23 & 359 & 0 & 11 & 12 \\
Escape & 15 & 0 & 377 & 1 & 12 \\
Jump & 27 & 5 & 3 & 214 & 21 \\
Do gymnastics & 53 & 2 & 17 & 13 & 185 \\
\hline
\end{tabular}

injury. All the heads were called to return to the same equation with the superiority, and the other 15 subjects were used as the verification group. The linear regression equation of energy expenditure was constructed by using the training data of 30 people, and the training data of the remaining 15 people were used to verify the regression equation. There was no high-intensity activity before 24 hours and no dietary intake before 1 hour.

(2) Experimental Method. Before the beginning of the experiment, in order to avoid affecting the fitting results of energy consumption detection equation due to the sequence of actions, the sequence of actions that each experimenter needs to complete in the experimental group is disturbed. During the experiment, the experimenter followed the voice and action guidance video to complete four kinds of fitness actions, including opening and closing jump, left and right small jump, squat, and skipping rope. The completion time of each action is 1 minute, and the next action is started after 3 minutes of rest. During the rest of the experimenter, the staff recorded the detection data of each action for subsequent analysis.

The experimental scheme of the experimenters in the verification group is the same as that in the experimental group. In addition, in order to compare the accuracy of energy consumption detection model used in daily physical activities with that constructed in special sports activities experiments, the experimenters in the verification group need to fix a mobile phone device of the same model in the right pocket of trousers to record the motion acceleration information collected by the mobile phone acceleration sensor.

(3) Data Statistics. After completing the designed experimental program, the experimenter uses the exercise cardiopulmonary function instrument to record the data in the exercise process for calculation and gets the energy consumption value of each experimenter. This energy consumption value includes exercise energy consumption and basal metabolism. In order to build a physical activity energy consumption detection model, it is necessary to exclude basal metabolism energy consumption first, as shown in

Exercise energy consumption after correction = Energy consumption of indirect calorimetry - Basal metabolism during exercise time.

SPSS 20 software was used for statistical analysis, and the corrected energy consumption recorded by cardiopulmonary function instrument and the acceleration data calculated from image data were entered into the software in a one-to-one correspondence relationship. Pearson analysis was used to analyze the correlation between standard energy consumption and acceleration, sex of experimenter, BMI, and other indexes, and stepwise regression method was used to build an energy consumption detection model. The Bland-Altman (B-A diagram) analysis in MedCalc software 
TABLE 5: Basic information of experimenters.

\begin{tabular}{lcc}
\hline Indicators & Male $(n=35)$ & Female $(n=10)$ \\
\hline Age (years) & $25.2 \pm 5.2$ & $24.6 \pm 3.8$ \\
Height $(\mathrm{cm})$ & $182.1 \pm 5.2$ & $161.2 \pm 3.8$ \\
Body weight $(\mathrm{kg})$ & $80.5 \pm 7.3$ & $48.7 \pm 5.6$ \\
BMI $\left(\mathrm{kg} / \mathrm{m}^{2}\right)$ & $24.3 \pm 3.4$ & $18.9 \pm 2.8$ \\
\hline
\end{tabular}

is used to verify the consistency between the predicted value and the standard value, and the prediction accuracy of the energy consumption detection model is evaluated by calculating RMSE and MAPE coefficients of the predicted value and the standard value.

4.2.2. Establishment of Energy Consumption Model. In the energy consumption experiment of special sports activities, the acceleration values used include the activity information of all key body parts. Therefore, in the construction of energy consumption model of special sports activities, there is no need to build energy consumption detection models according to sports intensity, and the sports acceleration data and standard energy consumption data of the experimental group are only processed for different genders to build corresponding comprehensive energy consumption detection models.

Table 6 is a nested energy consumption model based on male VM value, in which Model 1 is a basic energy consumption model only considering VM value and $R$ is 0.62 . Compared with Model 1, Model 2 with BMI index has improved the prediction accuracy of the model to 0.65 , so the comprehensive energy consumption detection model based on male VM value is shown in

$\frac{\text { Energy consumption }}{5 \mathrm{~s}}=0.000525 \times \mathrm{VM}+0.0087 \times \mathrm{BMI}=0.261$.

Table 7 is a nested energy consumption model based on women's VM value, in which Model 1 is a basic energy consumption model only considering VM value and $R$ is 0.67. Compared with Model 1, Model 2 with BMI index has improved the prediction accuracy of the model, and $R$ is increased to 0.70 . Therefore, a comprehensive energy consumption detection model based on women's VM value is shown in

$\frac{\text { Energy consumption }}{5 \mathrm{~s}}=0.000589 \times \mathrm{VM}+0.046 \times \mathrm{BMI}-0.581$.

4.2.3. Band-Back Verification of Energy Consumption Model. The acceleration VM values and BMI values of 15 experimenters in the verification group were brought into the corresponding energy consumption detection model, the predicted values of the model were obtained, and the synchronous standard values were recorded. The Bland-Altman statistical method (B-A diagram) in MedCalc software is used to verify the consistency between the standard energy consumption
TABLE 6: Energy nesting model (based on male VM value).

\begin{tabular}{lcc}
\hline & $\begin{array}{c}\text { Model 1 } \\
\text { Energy consumption }\end{array}$ & $\begin{array}{c}\text { Model 2 } \\
\text { Energy consumption }\end{array}$ \\
\hline \multirow{2}{*}{$\mathrm{VM}$} & 0.000532 & 0.000525 \\
& 0.000 & 0.000 \\
\hline \multirow{2}{*}{ BMI } & & 0.0087 \\
& & 0.005 \\
\hline \multirow{2}{*}{ Constant } & 0.45 & 0.261 \\
& 0.000 & 0.000 \\
\hline \multirow{2}{*}{$R^{2}$} & 0.62 & 0.65 \\
& 0.000 & 0.000 \\
\hline
\end{tabular}

TABLE 7: Energy nesting model (based on female VM value).

\begin{tabular}{lcc}
\hline & Model 1 & Model 2 \\
& Energy consumption & Energy consumption \\
\hline \multirow{2}{*}{ VM } & 0.000638 & 0.000589 \\
& 0.000 & 0.000 \\
\hline \multirow{2}{*}{ BMI } & & 0.046 \\
& & 0.000 \\
\hline \multirow{2}{*}{ Constant } & 0.345 & -0.581 \\
& 0.000 & 0.000 \\
\multirow{2}{*}{$R^{2}$} & 0.67 & 0.70 \\
& 0.000 & 0.000 \\
\hline
\end{tabular}

value and the predicted energy consumption value, and the deviation confidence interval of the B-A diagram is $95 \%$.

As shown in Figure 5, in the B-A graph of men in the verification group, 7 points fall outside $1.96 \mathrm{SD}$, accounting for $3.5 \%$ of the total data volume, and in the B-A graph of women in the verification group, 9 points fall outside 1.96 $\mathrm{SD}$, accounting for $4.5 \%$ of the total data volume, all of which do not exceed the 5\% standard. In addition, the average difference between standard and forecast is 0.00 and -0.02 , respectively, which is close to the mean line 0 . Therefore, it is considered that the two comprehensive energy consumption detection models constructed by experiments have strong prediction ability.

4.2.4. Comparison of Accuracy of Different Energy Consumption Models. VM values and BMI values collected by mobile phone acceleration sensors worn by 15 experimenters in the verification group were brought into the daily physical activity energy consumption detection model to obtain the energy consumption prediction value of the daily physical activity model, and at the same time, the energy consumption prediction values collected by the special sports activity energy consumption detection model were recorded. The RMSE and MAPE indexes of the predicted values and standard values of the two models are used to evaluate the prediction accuracy of the models, and the calculation methods are shown in formulas (32) and 

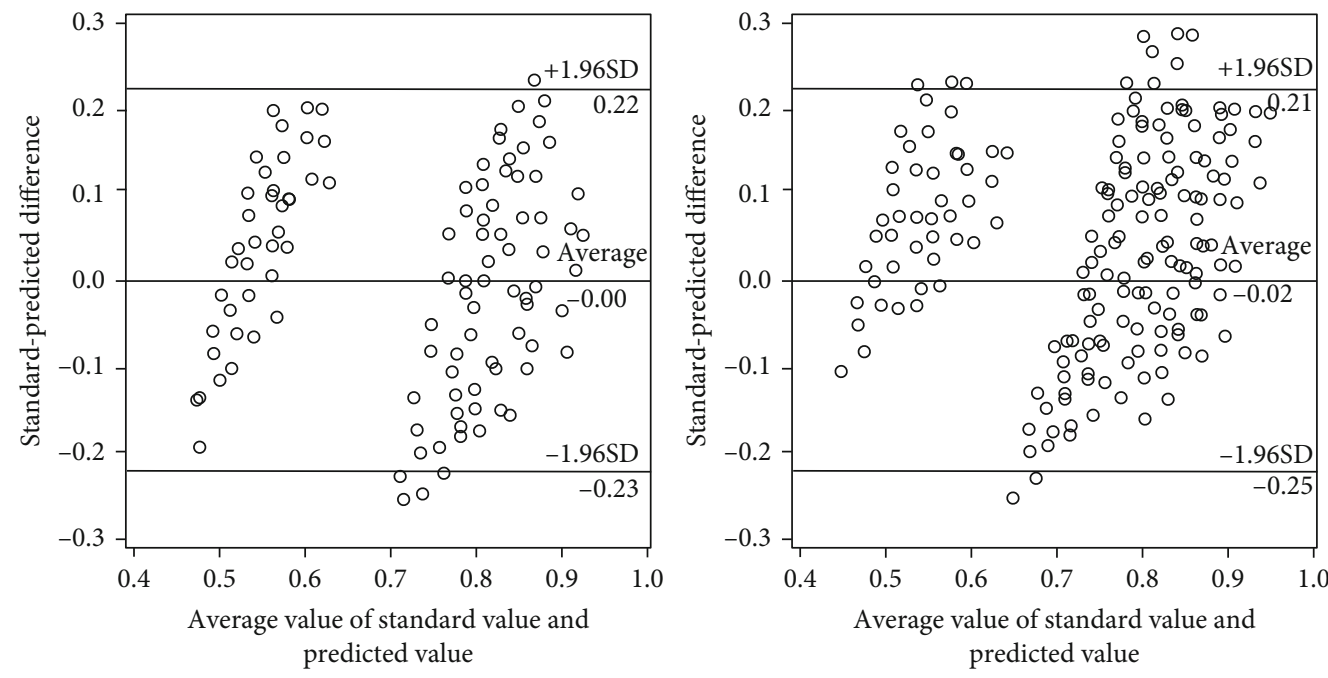

Figure 5: B-A diagram of (a) men and (b) women.
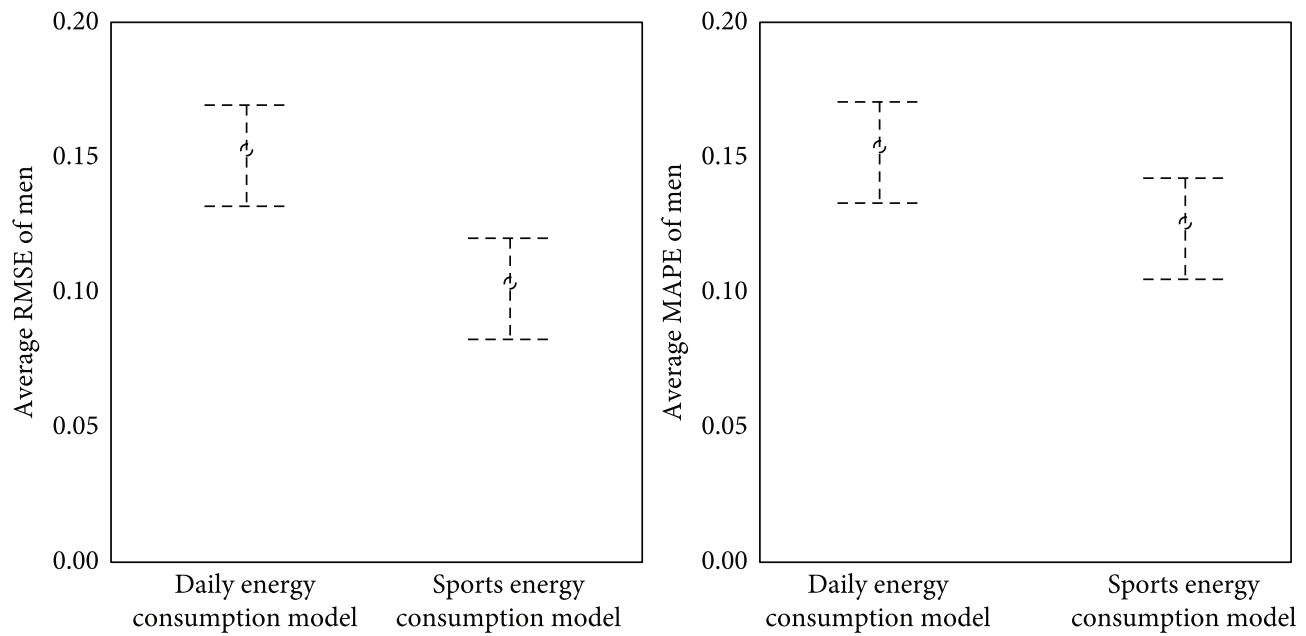

FIgure 6: Comparison of indicators of male energy consumption detection models.
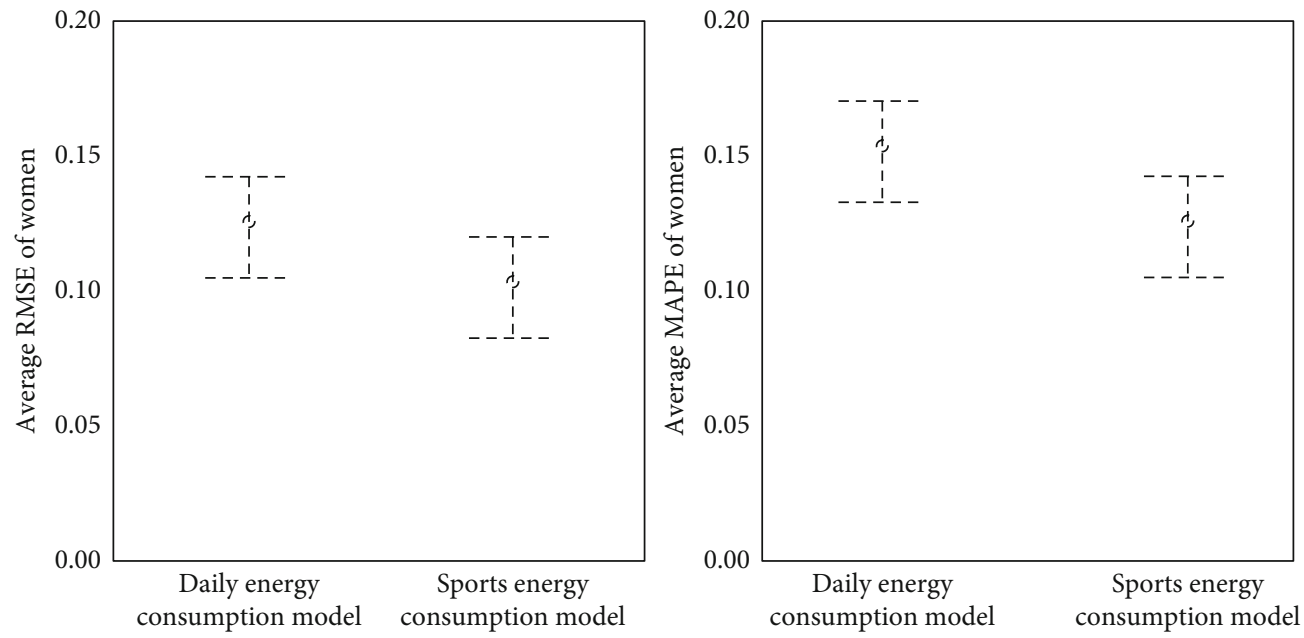

FIGURE 7: Comparison of indicators of women's energy consumption examination model. 
(33). RMSE is the root mean square difference between standard and prediction, and the smaller RMSE, the higher the prediction accuracy of the model; MAPE is the average percentage difference between the standard and the forecast, and it is usually recognized that the MAPE value is less than $10 \%$, which indicates that the accuracy of the model is high.

$$
\begin{gathered}
\text { RMSE }=\sqrt{\frac{\sum(\text { Predicted value }- \text { standard value })^{2}}{N},} \\
\text { MAPE }=\sum \frac{\mid \text { Predicted value }- \text { standard value } \mid}{\text { Standard value }} \times \frac{100}{N} .
\end{gathered}
$$

Figures 6 and 7 are the comparison charts of RMSE and MAPE indexes of two energy consumption detection models for men and women. It can be seen from the figure that RMSE and MAPE values of energy consumption detection model for special sports activities are lower than those of daily physical activity model. In the comparison of male energy consumption models, RMSE value of special sports energy consumption model is $0.12+0.03$, and the MAPE value is $6.45 \pm 0.72 \%$. The RMSE value of daily physical activity energy consumption model is $0.15 \pm 0.04$, and the MAPE value is $7.93 \pm 0.726 \%$. In the comparison of female energy consumption models, the RMSE value of special sports energy consumption model is $0.12 \pm 0.04$, and the MAPE value is $8.09 \pm 1.09 \%$. The RMSE value and MAPE value of daily physical activity energy consumption model were $0.13 \pm 0.04$ and $9.63 \pm 1.01 \%$, respectively. From the above RMSE value, we can see that the average value of men and women is less than 0.2 , which indicates that the accuracy of energy consumption model is better. However, the average MAPE value of men and women is less than 0.1 , which indicates that the accuracy of energy consumption model is high. From the analysis of the overall index value, it is concluded that the model constructed in this experiment has higher prediction accuracy.

4.3. Experimental Analysis. According to the comprehensive analysis of the data during the exercise, there is a significant correlation between sports energy consumption and morphological indexes such as gender, height, weight, and BMI. BMI is calculated from height and weight, while gender is used as classification index, and energy consumption between different genders is analyzed separately. Therefore, gender and BMI are considered as independent variables in the model construction. Under the same age and exercise type, men's activity energy consumption is higher than that of women, which is in line with the actual research conclusion: there are differences in fat situation and metabolic level between men and women, which leads to differences in energy consumption, so it is reasonable to include gender and BMI as independent variables in the construction of energy consumption detection model.
The comprehensive energy consumption detection model based on VM values of men and women is shown in

Male $: \frac{\text { Energy consumption }}{5 \mathrm{~s}}=0.000525 \times \mathrm{VM}+0.0087 \times \mathrm{BMI}+0.261$,

Female $: \frac{\text { Energy consumption }}{5 \mathrm{~s}}=0.000589 \times \mathrm{VM}+0.046 \times \mathrm{BMI}+0.581$

After adopting stepwise regression method, the BMI index is brought into the energy consumption detection model, and the correlation between the predicted results and the standard values is increased from 0.62 to 0.65 and 0.67 to 0.70 , respectively, which makes the interpretation of the model higher.

Figure 5 is the verification result of the energy consumption detection model of special sports activities. From the BA diagram corresponding to male and female, it can be seen that the difference between the standard value and the predicted value of energy consumption of the experimenters in the verification group mostly falls within $1.96 \mathrm{SD}$, and the number of points falling outside the range is less than $5 \%$. The reasons for this situation include the following:

(1) Considering that the data collected by mobile phone equipment and the data collected by exercise cardiopulmonary instrument are manually synchronized by the staff, there may be inconsistent data

(2) In the aspect of data processing, although the original skeleton point data is screened and processed, there may still be errors caused by skeleton point data shaking or disappearing

(3) The original data filtering algorithm is relatively simple, which may filter out some useful skeleton point information, resulting in some errors in the final energy consumption detection model verification results

This kind of error is within the acceptable range of experimental results, and the final energy consumption detection model is reliable. In order to verify the superiority of the energy consumption detection method and the constructed model of special sports activities, RMSE and MAPE indexes are used to verify the two energy consumption detection models of daily physical activity and special sports activities. The results are shown in Figures 6 and 7. The analysis results from the two energy consumption models in different genders are as follows. The prediction accuracy of the model constructed in this experiment is higher. The future work mainly studies the correlation and prediction of human body data perceived by human body multisensors, and the difference memory connection of different data can find the predictability of human body monitoring data as early as possible.

\section{Conclusion}

The basic metabolism model and energy supply and demand model of the human body were analyzed, and a scientific 
energy consumption test model of special sports activities was constructed. An algorithm of heart rate detection and energy consumption based on acceleration data acquisition is proposed. Therefore, it is proposed to use the bone point data obtained by portable telephone camera sensor to calculate the motion acceleration and calculate the kinetic energy consumption through the detected data. A model for evaluating the proposed algorithm is also established, which can accurately collect human heart rate and energy consumption through sensor sensing information, thus providing health monitoring and further analyzing human health.

\section{Data Availability}

The experimental data used to support the findings of this study are available from the corresponding author upon request.

\section{Conflicts of Interest}

The authors declared that they have no conflicts of interest regarding this work.

\section{References}

[1] J. Geisheimer and E. Greneker, "A non-contact lie detector using radar vital signs monitor (RVSM) technology," IEEE Aerospace \& Electronics Systems Magazine, vol. 16, no. 8, pp. 10-14, 2001.

[2] I. Waltering and G. Sl, "Identification of factors for a successful implementation of medication reviews in community pharmacies: using positive deviance in pharmaceutical care," International Journal of Clinical Pharmacy, pp. 1-11, 2021.

[3] A. Podolskij, "Reprint of: The system of planned, stage-bystage formation of mental actions (PSFMA) as a creative design of psychological conditions for instruction," Learning Culture and Social Interaction, vol. 27, no. 4, p. 100474, 2020.

[4] V. Cantino, E. Giacosa, S. Alfiero, S. M. R. Shams, and A. Ferraris, "Introduction: smart tourism businesses (sustainability, measurability, awareness, recognition, \& technology)," Tourism Analysis, vol. 24, no. 3, pp. 261-263, 2019.

[5] Y. Nie, K. Xiao, and J. He, "Exploring the cultivation path of public information literacy under the "information epidemic situation" (in Chinese)," Shanxi Archives, vol. 2, pp. 10-18, 2020.

[6] Y. Xiao, "Feasibility study of tabata training in the reform of physical education teaching in colleges and universities," E3S Web of Conferences, vol. 236, article 04022, 2021.

[7] J. Liu and D. Y. Huang, "Interpretation of the paths used in aerobics in colleges and universities under the background of big data," Journal of Physics: Conference Series, vol. 1574, no. 1, article 012118, 2020.

[8] A. A. Akhmatgatin, V. Y. Lebedinsky, G. K. Khomyakov et al., "The effectiveness of students' preparation in physical training at the universities of various departments," Physical Education of Students, vol. 24, no. 1, pp. 4-10, 2020.

[9] A. Rapp, "Design fictions for learning: a method for supporting students in reflecting on technology in human-computer interaction courses," Computers \& Education, vol. 145, article 103725, 2020.
[10] J. Xiao, J. Qu, and Y. Li, “An electrooculogram-based interaction method and its music-on-demand application in a virtual reality environment," IEEE Access, vol. 7, pp. 22059-22070, 2019.

[11] C. Yang and H. Ming, "Detection of sports energy consumption based on IoTs and cloud computing," Sustainable Energy Technologies and Assessments, vol. 46, no. 3, p. 101224, 2021.

[12] M. Mason, "The elements of visitor experience in post-digital museum design," Design Principles and Practices: An International Journal-Annual Review, vol. 14, no. 1, pp. 1-14, 2020. 